\section{Emerging Leptospirosis, North India}

To the Editor: We read with interest the article, The Changing Epidemiology of Leptospirosis in Israel, published in volume 7, no. 6 (1). Leptospirosis, a septicemic zoonosis with multisystemic involvement, is caused by the pathogenic strains of Leptospira interrogans. Rural farm workers are at high risk for leptospirosis, and it can be a significant public health problem when water and food safety are not ensured. Several epidemics of leptospirosis have occurred on Andaman and Nicobar islands and in southern and western parts of India during the past century (2). The organism has been detected in farm animals in many parts of the country (3); however, human infections have been more or less localized. In 1998, researchers warned that, unless adequate public health measures were initiated, large leptospirosis epidemics were possible in areas where the disease had not been previously reported (4). In addition, they recommended improving clinical diagnosis and conducting systematic epidemiologic studies for control of the disease (4).

The true incidence of human leptospirosis in northern India is not known either because of a lack of awareness on the part of the treating physicians or the lack of diagnostic techniques. In 1966, human leptospirosis was reported in Delhi, a state in northern India (5). In a 1966 study (5), sera from persons with pyrexia and jaundice were tested by the agglutination lysis test for leptospiral antibodies. Of 93 serum specimens from persons with pyrexia cases, 3 were positive ( 1 with $L$. icterohemorrhagica and two with L. canicola); of 43 serum specimens from persons with jaundice, 3 were positive ( 2 with $L$. icterohemorrhagica and 1 with $L$. icterohemorrhagica and L. pomona). No other study on leptospirosis has been done in the region, and no data are available concerning the problem.

To assess the current status of transmission in Delhi and its adjoining areas, we conducted a systematic study for the diagnosis of leptospirosis in our hospital from April 2000 to March 2001; case definition criteria suggested in a previous study (4) were used. A case was defined as a person with fever, headache, and myalgia and more than two of the following symptoms: jaundice, oliguria, respiratory symptoms (cough, hemoptysis, and breathlessness), hemorrhagic manifestations (hematemesis, bleeding gums, and subconjunctival hemorrhage), and signs of meningeal irritation and convulsion. Seventy-five patients (44 male patients; $3-73$ years of age) satisfied the inclusion criteria. In addition to clinical evaluation and assessment for other diseases, leptospirosis was investigated by the following laboratory methods: isolation of Leptospira interrogans, direct visualization of the organism under dark-field microscopy, and enzyme-linked immunosorbent assay (ELISA) for Leptospira immunoglobulin (Ig) $\mathrm{M}$ antibody (Serion Immunodiagnostica $\mathrm{GmbH}$, Würzburg, Germany). Per manufacturer's specifications, the sensitivity, specificity, positive predictive value, and negative predictive value of this kit are $96 \%, 97 \%, 90 \%$, and $99 \%$, respectively). All blood samples were sent to the Leptospira referral laboratory at the Indian Veterinary Research Institute, Izzatnagar, for microscopic agglutination test (MAT). Eight serovars of $L$. interrogans (australis, autumnalis, pomona, sejroe, tarassovi, icterohaemorrhagica, hebdomadis, and patoc) were tested, and a agglutination titer of more than 1:100 was considered positive. All patients were treated empirically with broad-spectrum antibiotics as well as specific drugs according to the results of investigations.

Thirty-two patients (42.6\%) had a positive ELISA test for Leptospira IgM antibody. The results of MAT were positive in $21(65.6 \%)$ of the 32
ELISA-positive serum samples. Serum specimens from 11 patients reacted with a single serovar, and specimens from 10 patients reacted with more than one serovar. Among the pathogenic species, Leptospira antibodies were detectable by MAT predominantly against L. sejroe ( 7 of 21), followed by L. icterohaemorrhagica (6 of 21), L. hebdomadis (4 of 21), and L. tarassovi (4 of 21). Leptospira antibodies were also detectable against L. autumnalis (3 of 21), L. australis ( 2 of 21), and L. pomona (1 of 21). Against L. patoc, MAT could detect antibodies in six samples. The organism could not be isolated in culture or visualized under dark-field microscopy in any of the specimens. Of the 43 case-patients with ELISAnegative specimens, alternative diagnoses were established for 40 on the basis of various laboratory investigations. In five of the patients with ELISA-positive specimens, coinfection with other pathogens was detected, including Salmonella typhi (one case) by a positive Widal test, hepatitis $\mathrm{C}$ virus by positive ELISA (two cases), and Plasmodium falciparum (two cases) by a positive smear. Five patients, including three who were ELISA positive for Leptospira, died. The highest number of ELISApositive serum samples (21 of 32) were obtained in August and September 2000, suggesting an epidemic.

Epidemiologic investigation of leptospirosis is often hampered by the difficulty of making a definitive microbiologic diagnosis. Isolation of leptospira from clinical samples provides a definitive diagnosis; however, the value of culture is limited because samples have to be collected before the administration of antibiotics, and culturing requires prolonged incubation. Demonstration of typical motility of leptospira under dark-ground illumination in clinical samples, though helpful in early diagnosis, has low sensitivity and depends on the technician's opinion. Measurement of IgM antibodies against Leptospira by ELISA has emerged as a reliable diag- 
nostic test with good specificity and sensitivity (6). The probability of achieving a positive serologic test increases with the duration of disease, and good correlation between results of MAT and ELISA has been reported by Cumberland et al. (7). MAT has emerged as a dependable diagnostic tool for leptospirosis (next to isolation) by providing serovar specific diagnosis. However, a large number of serovars of $L$. interrogans exist, and maintaining large numbers of organisms for MAT is difficult for most laboratories. Moreover, MAT may fail to detect antibodies when specific serovars are not used. In this study, the ELISA-positive samples, for which MAT results were negative, may have been caused by infection with serovars other than those used in this study. Because of the problems with methods, leptospirosis is grossly underdiagnosed.

Leptospira organisms require humid weather for their survival. Rodents and domestic animals (i.e., cattle and dogs) harbor leptospires and shed the bacteria in urine; they may disseminate the organism to rain water and drinking water sources. Humans frequently come in contact with contaminated water during floods; the number of cases is higher during and after heavy rainfalls. We found that the peak incidence of the disease was during August and September, the monsoon season, which may explain the high incidence of seropositivity during this period. Though the organism has been detected in farm animals in northern India, human leptospirosis has not been considered a major public health problem, probably because transmission is low in arid weather conditions. As a result of 13 consecutive monsoons of above-average strength in India, changes in the environment may be promoting the transmission of this organism. Recently, two other regions in northern India, Chandigarh (8) and Varanasi (9), have reported a Leptospira seroprevalance rate of $8.8 \%$ and $21.74 \%$, respectively.
Our study supports the warning from other researchers regarding the threat of leptospirosis in areas such as northern India. Preventive measures should be initiated and rapid and definitive diagnostic tests must be developed.

\section{Rama Chaudhry*, M.M. Premlatha, ${ }^{*}$ Srujana Mohanty, ${ }^{*}$ Benu Dhawan, ${ }^{*}$ Kumar Kirti Singh, ${ }^{*}$ and A.B Dey*}

*All India Institute of Medical Sciences, New Delhi, India

\section{References}

1. Kariv R, Klempfner R, Barnea A, Sidi Y, Schwartz E. The changing epidemiology of leptospirosis in Israel. Emerg Infect Dis 2001;7:990-2.

2. Sehgal SC. Leptospirosis in the horizon. Natl Med J India 2000;13:228-30.

3. Ratnam S. Leptospirosis: an Indian perspective. Indian Journal of Medical Microbiology 1994;12:228-39.

4. Singhal RL, Sood OP, editors. Leptospirosis. Proceedings of the Third Round Table Conference; 1998 Feb 23; New Delhi, India. Gugaon, India: Ranbaxy Science Foundation; 1998.

5. Joseph KM, Kalra SL. Leptospirosis in India. Indian J Med Res 1966;54:611-4

6. Winslow WE, Merry DJ, Pirc ML, Devine PL. Evaluation of a commercial enzyme- linked immunosorbent assay for detection of immunoglobulin $\mathrm{M}$ antibody in diagnosis of human leptospiral infection. J Clin Microbiol 1997;35:1938-42.

7. Cumberland P, Everard CO, Levett PN. Assessment of the efficacy of an IgMELISA and microscopic agglutination test (MAT) in the diagnosis of acute leptospirosis. Am J Trop Med Hyg 1999;61:731-4.

8. Pooja, Sharma M, Sud A, Sethi S. Serological evidence of leptospirosis by IgM ELISA and IgM dipstick in patients of acute febrile illness. In: XXVth National Congress of Indian Association of Medical Microbiologists; 2001 Nov 21-25; New Delhi, India. New Dehli, India: Organizing Committee of the XXVth National Congress of the Indian Association of Medical Microbiologists; 2001. p. 90.

9. Kumar D, Tripathi K, Mohapatra TM. Detection of leptospirosis for the first time in the eastern zone of northern India: a preliminary report. In: XXVth National Congress of Indian Association of Medical Microbiologists; 2001 Nov 21-25; New Delhi, India New Delhi, India: Organizing Committee of the XXVth National Congress of the Indian Association of Medical Microbiologists; 2001.p. 90.

Address for correspondence: Rama Chaudhry, All India Institute of Medical Sciences, New Delhi, 110029, India; fax: 91-11-6862663; e-mail: rc123@hotmail.com

\section{James A. Ferguson Emerging Infectious Diseases Fellowship}

The Office of Minority and Women's Health, National Center for Infectious Diseases, Center for Disease Control and Prevention (CDC), announces the James A. Ferguson Emerging Infectious Diseases Fellowship Program, 2003.

This fellowship program is an 8-week professional development experience for racial and ethnic minority students in medical, dental, veterinary, pharmacy, and masters of public health graduate programs. Fellows participate in a broad array of public health activities. The program is administered through a cooperative agreement between the Minority Health Professions Foundation and the National Center for Infectious Diseases, CDC. Fellows are paired with a mentor based on their statement of interests and qualifications. They are required to prepare and present a formal scientific presentation on their work to CDC scientists and staff at the end of the program and to submit a formal research paper. The students receive stipends, housing, and transportation to and from Atlanta.

The program is designed to increase the students' knowledge of public health and public health career paths and to introduce fellows to careers addressing infectious diseases and racial and ethnic health disparities. The ultimate goal of the program is to influence students to pursue careers in public health and specific disciplines needed by the National Center for Infectious Diseases to strengthen and diversify the workforce.

The deadline for submitting applications for this fellowship is February 28, 2003. For additional information about the program, please contact Edith A. Hambie at eah1@cdc.gov, or call 404-371-5310. 